\title{
Surface step states and Majorana end states in profiled topological-insulator thin films
}

\author{
Peter Thalmeier ${ }^{1}$ and Alireza Akbari $\oplus^{1,2}$ \\ ${ }^{1}$ Max Planck Institute for the Chemical Physics of Solids, D-01187 Dresden, Germany \\ ${ }^{2}$ Max Planck POSTECH Center for Complex Phase Materials and Department of Physics, POSTECH, Pohang, Gyeongbuk 790-784, Korea
}

(Received 5 August 2021; revised 29 October 2021; accepted 4 November 2021; published 18 November 2021)

\begin{abstract}
The protected helical surface states in thin films of topological insulators (TIs) are subject to intersurface hybridization. This leads to gap opening and spin texture changes as witnessed in photoemission and quasiparticle interference investigations. Theoretical studies show that universally, the hybridization energy exhibits exponential decay as well as sign oscillations as a function of film thickness, depending on the effective band parameters of the material. When a step is introduced in the TI film, e.g., by profiling the substrate such that the hybridization has different signs on both sides of the step, one-dimensional bound states appear within the hybridization gap which decay exponentially with distance from the step. The step bound states have linear dispersion and inherit the helical spin locking from the surface states and are therefore nondegenerate. When the substrate becomes an $s$-wave superconductor, Majorana zero modes located at the step ends are created inside the superconducting gap. The proposed scenario involves just a suitably stepped interface of a superconductor and a TI and therefore may be the simplest device able to host Majorana zero modes.
\end{abstract}

DOI: 10.1103/PhysRevB.104.184511

\section{INTRODUCTION}

The surfaces of strong topological insulators (TIs) like $\mathrm{Bi}_{2} \mathrm{Se}_{3}, \mathrm{Bi}_{2} \mathrm{Te}_{3}$, and $\mathrm{Sb}_{2} \mathrm{Te}_{3}$ carry spin-locked nondegenerate helical surface states. As long as the surfaces are isolated, i.e., their distance is much larger than the surface state decay length into the bulk, the two-dimensional (2D) dispersion is described by isotropic Dirac cones to lowest order in momentum counted from the time-reversal invariant (TRI) points and by warped cones with sixfold symmetry due to higher-order terms. They have been verified indirectly by magnetotransport measurements $[1,2]$ as well as directly by photoemission [3-6] and surface tunneling [7-13] experiments; the latter were explained in numerous theoretical investigations [13-15]. This simple situation changes in an interesting manner when one considers TI thin films with a thickness small enough that intersurface hybridization of bottom and top surface states occurs. Due to the interaction topological protection for states close to the Dirac point is lifted, and a hybridization gap in the excitation spectrum opens. This has, indeed, been verified directly by photoemission [16,17] and also by a sudden breakdown of weak antilocalization in magnetotransport $[1,2]$ as a function of film thickness when the latter falls below about five quintuple layers (QLs). The effective hybridization between the surface states has been calculated [18-20] by solving the thin-film

Published by the American Physical Society under the terms of the Creative Commons Attribution 4.0 International license. Further distribution of this work must maintain attribution to the author(s) and the published article's title, journal citation, and DOI. Open access publication funded by the Max Planck Society. boundary value problem for an effective $\mathbf{k} \cdot \mathbf{p}$ Hamiltonian of the bulk. Due to the constrained film geometry the effective intersurface hybridization $t(d)$ not only decreases exponentially with film thickness $d$ but also generally oscillates as a function of thickness $d$ with an oscillation period depending on the material parameters. This should again be visible in the photoemission gap and in the concomitant oscillation of quasiparticle interference (QPI) patterns predicted in Ref. [21].

Aside from the modulated excitation gap at the Dirac point the oscillation of $t(d)$ gives rise to another novel and highly interesting scenario which is the subject of this investigation. Suppose the film thickness is not constant but changes in a steplike manner at a certain lateral position (see Fig. 1). If the thicknesses to the left $\left(d_{L}\right)$ and right $\left(d_{R}\right)$ of the step are chosen in such a way that the hybridizations $t_{L}\left(d_{L}\right)$ and $t_{R}\left(d_{R}\right)$ have opposite signs on the two sides of the step, then a helical nondegenerate bound state within the hybridization gap may appear which is spatially located at the step and has linear dispersion. If it were found experimentally, this would entail a further interesting speculative possibility: Once the substrate of the step-profiled TI becomes a simple $s$-wave spin singlet superconductor, the proximity effect will open a superconducting gap in the dispersion of the nondegenerate (spin-locked) step state. This is a typical situation that can create Majorana end states. Such scenarios were previously investigated, e.g., with TI nanowires on a superconducting (SC) substrate [22-27] which may need the application of a magnetic flux through the wire. In alternative devices the wire itself has to be ferromagnetic [28] or heterostructures with ferromagnetic layers are used [24]. In the present scenario no magnetic field has to be applied; a properly chosen step of the TI profile on the substrate is sufficient to create the possibility of Majorana states at the step ends. This seems 


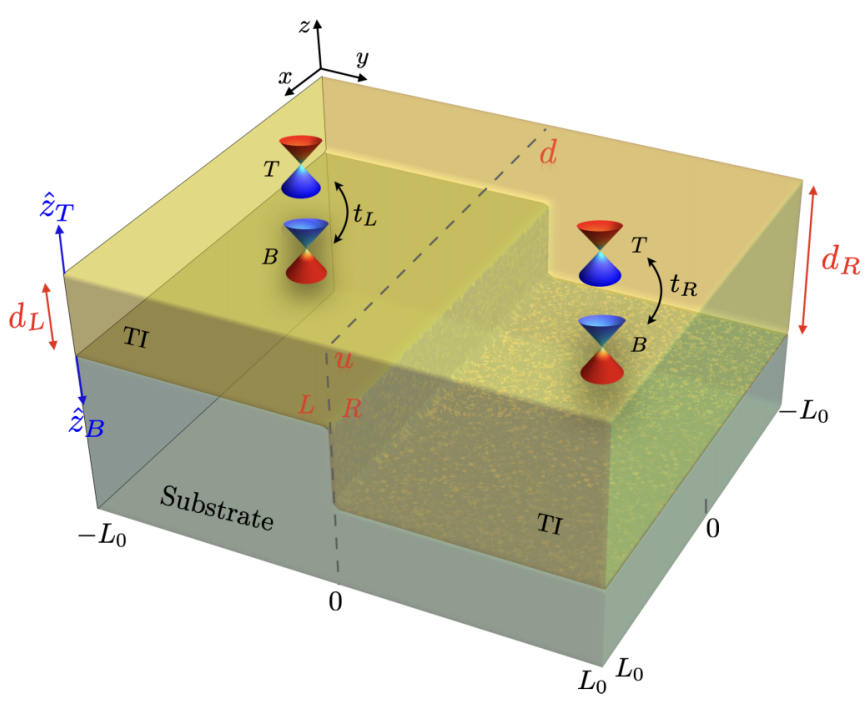

FIG. 1. Schematic view of the TI step configuration due to the profiled substrate; for simplicity the equal $x, y$ dimension $2 L_{0}$ of the device is chosen. The TI film thicknesses $d_{L}$ and $d_{R}$ to the left and right of the step lead to different hybridization strengths $t_{L}$ and $t_{R}$ between the Dirac cones on the top (T) and bottom (B) surfaces of the TI film. For $t_{L} t_{R}<0$ this leads to the appearance of a $1 \mathrm{D}$ bound state exponentially located at the step $y \approx 0$ and extended along $x$. If the substrate is an $s$-wave superconductor, Majorana zero modes may appear at the $\mathrm{u}$ and $\mathrm{d}$ positions. For clarity film thickness and step size are exaggerated.

to be a very simple way to realize these states in a realistic geometry.

\section{ISOTROPIC DIRAC SURFACE STATE MODEL FOR A TI}

As a starting point for the homogeneous thin film we use the isotropic TI surface state model on the top (T) and bottom (B) surfaces of the film. We will use $\alpha, \sigma$, and $\kappa$ indices and associated Pauli matrix vectors $(\boldsymbol{\alpha}, \boldsymbol{\sigma}, \boldsymbol{\kappa})$ to denote twodimensional T/B surface, $|\uparrow\rangle,|\downarrow\rangle$ spin, and $| \pm 1\rangle$ helicity spaces. The unit in each space is denoted by $\left(\alpha_{0}, \sigma_{0}, \kappa_{0}\right)$. On a single isolated surface, using the operator basis $\psi_{\mathbf{k}}^{\dagger}=$ $\left(c_{\uparrow \mathbf{k}}^{\dagger}, c_{\downarrow \mathbf{k}}^{\dagger}\right)$, the isotropic 2D model Hamiltonian is given by

$\mathcal{H}=\sum_{\mathbf{k}} \psi_{\mathbf{k}}^{\dagger} h_{\mathbf{k}} \psi_{\mathbf{k}}, \quad h_{\mathbf{k}}=v(\mathbf{k} \times \boldsymbol{\sigma}) \cdot \hat{\mathbf{z}}=v\left(\begin{array}{cc}0 & -i k_{-} \\ i k_{+} & 0\end{array}\right)$,

where $\mathbf{k}=\left(k_{x}, k_{y}\right)$ is the wave vector counted from the TRI Dirac point, $\sigma$ is the spin, $\hat{\mathbf{z}}$ is the surface normal, and $v$ is the velocity; furthermore, $k_{ \pm}=k_{x} \pm i k_{y}$, and $k=|\mathbf{k}|=\left(k_{x}^{2}+\right.$ $\left.k_{y}^{2}\right)^{\frac{1}{2}}$. This expression is proportional to the helicity operator $\kappa_{\hat{\mathbf{k}}}=(\sigma \times \mathbf{k}) \cdot \hat{\mathbf{z}} / k$, namely, $h_{\mathbf{k}}=(v k) \kappa_{\hat{\mathbf{k}}}$. The eigenvalues or dispersions of the Dirac cone and associated eigenvectors in the spin basis $|\uparrow\rangle,|\downarrow\rangle$ are described by

$$
\epsilon_{\mathbf{k}}^{ \pm}= \pm(v k), \quad S_{\mathbf{k}}=\frac{1}{\sqrt{2}}\left(\begin{array}{cc}
1 & i e^{-i \theta_{\mathbf{k}}} \\
-i e^{i \theta_{\mathbf{k}}} & 1
\end{array}\right)
$$

where the columns of the unitary matrix $S_{\mathbf{k}}$ are the helical eigenstates $| \pm 1\rangle$. In this basis the helicity operator is simply represented by the Pauli matrix $\kappa_{\hat{\mathbf{k}}}=\kappa_{z}$. Furthermore, $\theta_{\mathbf{k}}=$ $\tan ^{-1}\left(k_{y} / k_{x}\right)$ is the azimuthal angle of the $\mathbf{k}$ vector.

\section{HYBRIDIZATION AND GAP OPENING OF SURFACE STATES IN TI THIN FILMS}

In a TI film with thickness $d$ much larger than the surface state decay length the top and bottom surfaces may be considered independent surface states. One has to keep in mind only that surface normals are oppositely oriented to the global $\hat{\mathbf{z}}$ direction, i.e., $\hat{\mathbf{z}}_{T}=-\hat{\mathbf{z}}_{B} \equiv \hat{\mathbf{z}}$. Therefore, helicities for both energies are also opposite on $\mathrm{T}$ and $\mathrm{B}$ surfaces according to $\kappa_{\hat{\mathbf{k}}}^{B}=-\kappa_{\hat{\mathbf{k}}}^{T}$, and therefore, $h_{\mathbf{k}}^{B}=-h_{\mathbf{k}}^{T} \equiv-h_{\mathbf{k}}$. However, when the film thickness is reduced (below a few quintuple layers), the $\mathrm{T}$ and $\mathrm{B}$ surface states overlap, and a hybridization of equal-spin (and therefore equal-helicity) eigenstates develops. This problem has been fundamentally treated and analyzed in great generality in the work of Asmar et al. [19] and also in Ref. [29]. Here we use a simplified model with a k-independent effective hybridization element $t(d)$ that depends, however, on film thickness $d$. The thin-film surface state Hamiltonian is then given in the spin representation [21] using $\Psi_{\mathbf{k}}^{\dagger}=\left(c_{\uparrow \mathbf{k}}^{T \dagger}, c_{\downarrow \mathbf{k}}^{T \dagger}, c_{\uparrow \mathbf{k}}^{B^{\dagger}}, c_{\downarrow \mathbf{k}}^{B^{\dagger}}\right)$,

$$
\begin{aligned}
\mathcal{H} & =\sum_{\mathbf{k}} \Psi_{\mathbf{k}}^{\dagger} \hat{h}_{\mathbf{k}} \Psi_{\mathbf{k}}, \\
\hat{h}_{\mathbf{k}} & =v\left(k_{x} \sigma_{y}-k_{y} \sigma_{x}\right) \alpha_{z}+t \sigma_{0} \alpha_{x} \\
& =\left(\begin{array}{cc}
v\left(k_{x} \sigma_{y}-k_{y} \sigma_{x}\right) & t \sigma_{0} \\
t \sigma_{0} & -v\left(k_{x} \sigma_{y}-k_{y} \sigma_{x}\right)
\end{array}\right),
\end{aligned}
$$

or in the helicity representation according to

$$
\hat{h}_{\mathbf{k}}=\epsilon_{\mathbf{k}} \kappa_{z} \alpha_{z}+t \kappa_{0} \alpha_{x}=\left(\begin{array}{cc}
\epsilon_{\mathbf{k}} \kappa_{z} & t \kappa_{0} \\
t \kappa_{0} & -\epsilon_{\mathbf{k}} \kappa_{z}
\end{array}\right) .
$$

The eigenvalues of the film's Hamiltonian are then obtained as

$$
E_{\mathbf{k}}^{ \pm}= \pm\left[(v k)^{2}+t(d)^{2}\right]^{\frac{1}{2}}
$$

which exhibit a thickness-dependent hybridization gap $t(d)$ at the Dirac point $\mathbf{k}=0$. Each of these dispersion branches is twofold degenerate, which is inherited from the (T, B) degeneracy of states in the uncoupled $(t=0)$ case. The degeneracy is lifted if the bottom surface experiences an effective bias due to the substrate effect. This can be described by adding the term $\Delta_{s u} \sigma_{0} \alpha_{z}$ to Eq. (3). Then the split hybridized surface bands are given by

$$
E_{\mathbf{k} 1,2}^{ \pm}= \pm\left[\left(|v k| \pm\left|\Delta_{s u}\right|\right)^{2}+t(d)^{2}\right]^{\frac{1}{2}}
$$

where split band indices 1 and 2 refer to \pm inside the square root. Since the above substrate term can, in principle, be canceled by an applied bias voltage $(\mathrm{eV})$ term at the substrate, we will keep the degenerate thin film model of Eq. (5). Furthermore the chemical potential $\mu$ can be controlled by applying a gate voltage at the TI surface.

\section{A. The oscillation model of hybridization with film thickness}

The thickness-dependent effective hybridization $t(d)$ may be obtained from the solution of a subtle boundary value problem for the thin film [19], starting from the $\mathbf{k} \cdot \mathbf{p}$ Hamiltonian 
of the bulk bands. It may be represented by the phenomenological form $[19,21]$

$$
t(d)=t_{0} \exp \left(-\frac{d}{d_{0}}\right) \sin \left(\frac{d}{d_{0}^{\prime}}\right)
$$

Here the energy $t_{0}$ and thickness $\left(d_{0}, d_{0}^{\prime}\right)$ scales are determined by the parameters of bulk bands $[19,21]$. As an example we give the theoretical values for $\mathrm{Bi}_{2} \mathrm{Te}_{3}$ in terms of natural units $E^{*}=0.25 \mathrm{meV}$ and $1 \mathrm{QL}=10.16 \AA$, respectively, as $\left(t_{0}, d_{0}, d_{0}^{\prime}\right)=(0.80,1.79,0.3)$. As expected, the expression contains an exponential decay with increasing thickness $d$ but, in order to satisfy boundary conditions, also contains an oscillatory term whose physical origin was derived in Ref. [19]. The hybridization is obtained as a perturbation integral of an effective intersurface tunneling Hamiltonian connecting the uncoupled surface state wave functions. The decay length of the latter perpendicular to the surface may become a complex number, depending on the bulk band parameters. This leads to oscillating decay of the wave function which is inherited by the hybridization integral. We note that the vanishing $t(d)$ for thin films at the nodes (Fig. 2) does not mean the surface states should be considered decoupled as for large $d$ in the bulk case, but they rather indicate the destructive interference of both surface wave functions in the hybridization integral. These thickness oscillations of $t(d)$ play an essential role in the present investigation, and their consequences were studied before in view of their influence on QPI patterns [21]. According to the theoretical estimation of parameters $[19,20]$, the oscillations of the gap $|t(d)|$ should be pronounced in $\mathrm{Bi}_{2} \mathrm{Te}_{3}$ and $\mathrm{Sb}_{2} \mathrm{Te}_{3}$ but not in $\mathrm{Bi}_{2} \mathrm{Se}_{3}$. In the latter only half an oscillation period appears and is strongly damped by the rapid exponential decay of $t(d)$. The observable quantity is the (rectified) oscillation of the intersurface hybridization gap $|t(d)|$. Since it can reasonably be compared only for films with identical surface terminations, one is restricted to a discrete set of values for $t(d)$ with integer multiples of quintuple layers. Therefore, weak oscillations of $|t(d)|$ may not easily be identified. The hybridization gap opening as a function of film thickness with an integer number of QLs was investigated using angle-resolved photoemission spectroscopy (ARPES) [16] for $\mathrm{Bi}_{2} \mathrm{Se}_{3}$. The predicted exponential decay of $|t(d)|$ with increasing film thickness was observed, but no clear evidence of the half oscillation period was found. It was argued [21] that a modest change in the theoretical bulk band parameters leading to different values of $d_{0}$ and $d_{0}^{\prime}$ in Eq. (7) could account for the suppression of the half oscillation. In fact, charge transfer and bulk band bending effects due to the substrate have not been included in the theoretical model employed here but may influence the surface state energies [16] and possibly the above oscillation parameters. However, the origin of the hybridization gap oscillation has a universal nature enforced by boundary conditions due to the constrained geometry of thin films [19], and therefore, it may appear whenever the bulk band parameters of the three-dimensional (3D) TI lead to suitable $d_{0}$ and $d_{0}^{\prime}$ scales with $d_{0} \gg d_{0}^{\prime}$, i.e., a considerable number of oscillations before $t(d)$ decays. Therefore, they should appear in TI material with more favorable scale parameters such as those predicted for $\mathrm{Bi}_{2} \mathrm{Te}_{3}$ and $\mathrm{Sb}_{2} \mathrm{Te}_{3}$ [Fig. 2(a)]. So far no systematic film thickness variation of


FIG. 2. (a) Film thickness dependence of intersurface hybridization energy in $\mathrm{Bi}_{2} \mathrm{Te}_{3}$ for the theoretical model parameters $\left(t_{0}, d_{0}, d_{0}^{\prime}\right)=(0.8,1.79,0.3)$ corresponding to Ref. [19] (in units of $E^{*}=0.25 \mathrm{eV}$ for energy and QLs for thicknesses [21]). (b) Contour plot of the function $g\left(d_{L}, d_{R}\right)$ in Eq. (19). In the colored region a step bound state exists, while it is absent in the white regions. The darker colors correspond to more tightly bound wave functions in Fig. 4. The contour lines denote pairs $\left(d_{R}, d_{L}\right)$ with $t_{R}=-t_{L}$ leading to a symmetric step bound state around $y=0$. The green dots designate pairs with integer thickness (QL) close to this line of symmetry.

the hybridization gap in these materials has been investigated with either ARPES or QPI methods.

Due to their universal origin we are confident that the gap oscillations will eventually be found in thin films of a suitable TI material. This expectation is the starting point for the following investigation of the intriguing appearance of one-dimensional (1D) topological states for the profiled thin-film geometry.

\section{CREATION OF 1D STEP BOUND STATES INSIDE THE TI THIN FILM GAP}

The TI surface state forms linearly dispersing 2D bands or Dirac cones inside the bulk gap $\Delta_{b}$ [Fig. 3(a)] of the 3D TI material due to bulk spin-orbit coupling. For sufficiently thin films the Dirac cones themselves open a gap given by the size of the intersurface hybridization [Fig. 3(a)]. One might ask whether the creation of topologically protected in-gap states can be repeated by some means in a "matryoshka"- 


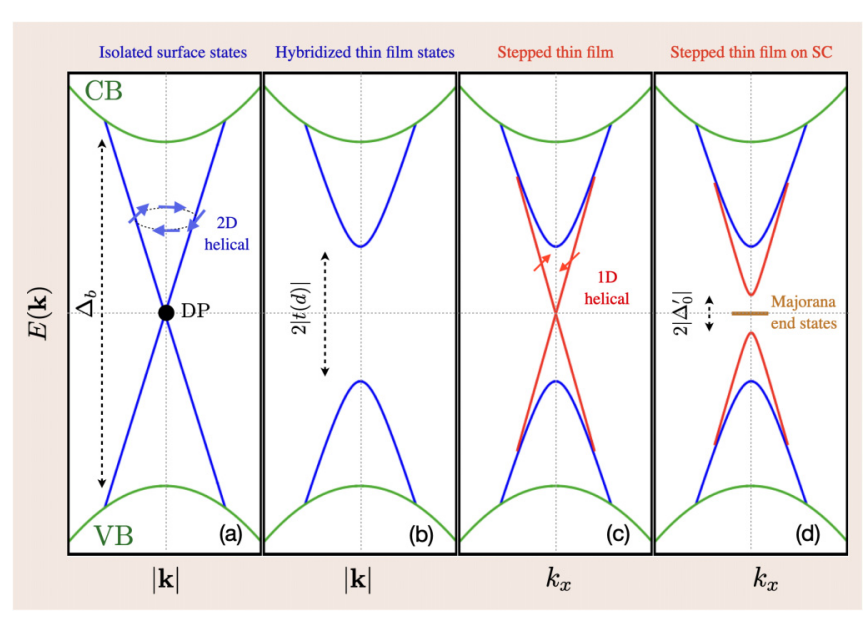

FIG. 3. Schematic sequence of 2D surface states and 1D step state gaps. (a) Ungapped 2D helical states on isolated surfaces. (b) Gapping in homogeneous thin films due to intersurface hybridization $t(d)$. (c) Appearance of 1D helical step states (red) in the profiled TI thin film with velocity $v$ asymptotically equal to that of $2 \mathrm{D}$ hybridized surface states (blue). (d) Opening of the SC proximity gap in the 1D step states and the appearance of zero-energy Majorana end states. Here $\Delta_{b}$ is the overall 3D bulk gap $\left(\Delta_{b} / E^{*} \simeq 1\right.$ for $\left.\mathrm{Bi}_{2} \mathrm{Te}_{3}\right)$. Note that for clarity the various gaps $\Delta_{b}, 2|t(d)|, 2\left|\Delta_{0}^{\prime}\right|$ are not drawn to scale.

like fashion, creating 1D linearly dispersing bands within the hybridization gap of $2 \mathrm{D}$ surface states. One way is to create domain walls of some sort on the surface with suitable properties. The easiest way to achieve a domain wall is by stepping the thin film (e.g., by stepping the substrate) so that the film thickness and hybridization are different on both sides of the step (see illustration in Fig. 1). This possibility will be investigated in the following sections.

\section{A. Boundary and existence conditions and dispersion of bound states}

Let us adopt a step geometry with the step extending along the $x$ direction, separating the left $(\mathrm{L})$ and right $(\mathrm{R})$ regions of the surfaces at $y=0$ and the surface normals oriented parallel to $z$ (Fig. 1). Then, assuming a sharp step, the $y$-dependent intersurface hybridization is given by

$$
t(y)=t_{L} \Theta(-y)+t_{R} \Theta(y),
$$

where $t_{L}=t\left(d_{L}\right)$ and $t_{R}=t\left(d_{R}\right)$ and we assume $2 L_{0}$ is the sample length along $x, y$ (Fig. 1). At the moment we keep the relative sizes of $d_{L}$ and $d_{R}$ arbitrary. To find out whether a localized state at the step develops one has to replace $k_{y} \rightarrow\left(-i \partial_{y}\right)$ and $t \rightarrow t(y)$ in the Hamiltonian leading to the effective 1D problem (with $k_{x}$ parallel to the step treated as a fixed parameter) described in spin-surface space by

$$
\begin{aligned}
\hat{h}\left(k_{x}, y\right) & =v\left(k_{x} \sigma_{y}+i \sigma_{x} \partial_{y}\right) \alpha_{z}+t(y) \sigma_{0} \alpha_{x} \\
& =\left(\begin{array}{cc}
v\left(k_{x} \sigma_{y}+i \sigma_{x} \partial_{y}\right) & t(y) \sigma_{0} \\
t(y) \sigma_{0} & -v\left(k_{x} \sigma_{y}+i \sigma_{x} \partial_{y}\right)
\end{array}\right) .
\end{aligned}
$$

If a localized bound state exists at the step, it has to fulfill the envelope equation

$$
\hat{h}\left(k_{x}, y\right) \boldsymbol{\phi}\left(k_{x}, y\right)=E \boldsymbol{\phi}\left(k_{x}, y\right),
$$

with $|E|<\left|t_{L}\right|,\left|t_{R}\right|$, i.e., lying inside the hybridization gap of $2 \mathrm{D}$ surface states. It is convenient to introduce rescaled energies $\hat{E}=E / v$ and $\hat{t}=t / v$, which have the dimension of the wave number or inverse length (units of $\mathrm{QL}^{-1}$ ). The wave function is a four-spinor defined by (tr indicates transposed)

$\boldsymbol{\phi}^{\mathrm{tr}}\left(k_{x}, y\right)=\left(\phi_{\uparrow}^{T}\left(k_{x}, y\right), \phi_{\downarrow}^{T}\left(k_{x}, y\right), \phi_{\uparrow}^{B}\left(k_{x}, y\right), \phi_{\downarrow}^{B}\left(k_{x}, y\right)\right)$.

For the the $\mathrm{L}$ and $\mathrm{R}$ sides of the step we use the following ansatz to solve Eq. (10) $(\lambda=L, R)$ :

$$
\phi_{\lambda}\left(k_{x}, y\right)=\mathbf{a}_{\lambda} e^{i k_{x} x} e^{i k_{y}^{\lambda} y}, \quad \mathbf{a}_{\lambda}^{\operatorname{tr}}=\left(a_{\uparrow}^{T \lambda}, a_{\downarrow}^{T \lambda}, a_{\uparrow}^{B \lambda}, a_{\downarrow}^{B \lambda}\right),
$$

where $k_{y}^{L}=-i \kappa_{L}$ and $k_{y}^{R}=i \kappa_{R}$ are given by the inverse decay lengths $\kappa_{R}$ and $\kappa_{L}$ of the bound state localized at the step. Inserting this into Eq. (10), we obtain

$$
\hat{E}^{2}=k_{x}^{2}-\kappa_{\lambda}^{2}+\hat{t}_{\lambda}^{2}, \quad \kappa_{L}^{2}-\kappa_{R}^{2}=\hat{t}_{L}^{2}-\hat{t}_{R}^{2} .
$$

The second equation follows because the first one has to be fulfilled for both sides, $\mathrm{L}$ and $\mathrm{R}$, simultaneously. The remaining relation to determine $\kappa_{\alpha}$ is obtained from the boundary condition at the step according to $\phi_{L}\left(k_{x}, 0\right)=\phi_{R}\left(k_{x}, 0\right)$. These $\lambda=L, R$ wave functions for step states with energies $\hat{E}$ [Eq. (13)] are determined by the solutions of

$$
\left(\begin{array}{cccc}
-\hat{E} & i\left(\kappa_{\lambda}-k_{x}\right) & \hat{t}_{\lambda} & 0 \\
i\left(\kappa_{\lambda}+k_{x}\right) & -\hat{E} & 0 & \hat{t}_{\lambda} \\
\hat{t}_{\lambda} & 0 & -\hat{E} & -i\left(\kappa_{\lambda}-k_{x}\right) \\
0 & \hat{t}_{\lambda} & -i\left(\kappa_{\lambda}+k_{x}\right) & -\hat{E}
\end{array}\right)\left(\begin{array}{c}
a_{\hat{\uparrow}}^{T \lambda} \\
a_{\downarrow}^{T \lambda} \\
a_{\hat{\hat{\lambda}}}^{B \lambda} \\
a_{\downarrow}^{B \lambda}
\end{array}\right)=0 .
$$

The matrix has rank 2, and therefore, two components, $a_{1} \equiv$ $a_{\uparrow}^{T \lambda}$ and $a_{2} \equiv a_{\downarrow}^{T \lambda}$, may be considered free parameters for the solution. The other two components are obtained as
The ratio $a_{1} / a_{2}$ is fixed by the continuity condition $\phi_{L}\left(k_{x}, 0\right)=\phi_{R}\left(k_{x}, 0\right)$. From the two equations above we obtain

$$
\begin{aligned}
& a_{B \uparrow}^{\lambda}=\frac{\hat{E}}{\hat{t}_{\lambda}} a_{1}-\frac{i\left(\kappa_{\lambda}-k_{x}\right)}{\hat{t}_{\lambda}} a_{2}, \\
& a_{B \downarrow}^{\lambda}=\frac{\hat{E}}{\hat{t}_{\lambda}} a_{2}-\frac{i\left(\kappa_{\lambda}+k_{x}\right)}{\hat{t}_{\lambda}} a_{1} .
\end{aligned}
$$

$$
\begin{aligned}
& \frac{a_{1}}{a_{2}}=i \frac{\hat{t}_{L}\left(\kappa_{R}+k_{x}\right)+\hat{t}_{R}\left(\kappa_{L}-k_{x}\right)}{\hat{E}\left(\hat{t}_{R}-\hat{t}_{L}\right)}, \\
& \frac{a_{2}}{a_{1}}=i \frac{\hat{t}_{L}\left(\kappa_{R}-k_{x}\right)+\hat{t}_{R}\left(\kappa_{L}+k_{x}\right)}{\hat{E}\left(\hat{t}_{R}-\hat{t}_{L}\right)} .
\end{aligned}
$$


Taking the product and using the symmetrized Eq. (13) with $\hat{E}^{2}=k_{x}^{2}-\frac{1}{2}\left[\left(\kappa_{R}^{2}+\kappa_{L}^{2}\right)-\left(\hat{t}_{R}^{2}+\hat{t}_{L}^{2}\right)\right]$, we finally arrive at the second relation

$$
\frac{1}{2}\left[\left(\kappa_{R}^{2}+\kappa_{L}^{2}\right)-\left(\hat{t}_{R}^{2}+\hat{t}_{L}^{2}\right)\right]=\left(\frac{\hat{t}_{L} \kappa_{R}+\hat{t}_{R} \kappa_{L}}{\hat{t}_{R}-\hat{t}_{L}}\right)^{2} .
$$

This equation together with the one in Eq. (13) may be solved by expressing them in terms of the (anti)symmetrized quantities $\frac{1}{2}\left(\kappa_{R} \pm \kappa_{L}\right)$. After some algebra one obtains the simple relations

$$
\begin{aligned}
& \kappa_{R}=\frac{1}{2}\left|\hat{t}_{R}-\hat{t}_{L}\right|+\frac{1}{2}\left(\hat{t}_{R}+\hat{t}_{L}\right) \operatorname{sgn}\left(\hat{t}_{R}-\hat{t}_{L}\right), \\
& \kappa_{L}=\frac{1}{2}\left|\hat{t}_{R}-\hat{t}_{L}\right|-\frac{1}{2}\left(\hat{t}_{R}+\hat{t}_{L}\right) \operatorname{sgn}\left(\hat{t}_{R}-\hat{t}_{L}\right) .
\end{aligned}
$$

For a localized step state within the thin-film hybridization gap one must have both $\kappa_{R}, \kappa_{L}>0$. It is easy to see from the above expressions that this is possible only if we have the fundamental relation



where we defined $\tau_{R L}=\operatorname{sgn}\left(\hat{t}_{R}-\hat{t}_{L}\right)$. Inserting $\kappa_{R, L}$ for these two cases into Eq. (13) for the step state energy we simply get, after reordering, the two linear dispersing energies:

$$
E_{k_{x} \pm}= \pm\left(v k_{x}\right)
$$

which form two linear dispersing branches of 1D excitations localized at and moving along the step with an energy inside the thin-film hybridization gaps for $\left|k_{x}\right|<\min \left\{\left|\hat{t}_{R}\right|,\left|\hat{t}_{L}\right|\right\}$. We note that the dispersion relation, i.e., the velocity $v$, is independent of the size of hybridizations $t_{L}$ and $t_{R}$ and hence of the associated asymmetric decay of the wave function perpendicular to the step. This nondegenerate step state as enforced by the boundary conditions exists as long as $\hat{t}(y)$ changes sign when crossing the step. It is therefore topologically protected as long as this condition is fulfilled. These 1D topological states inside the gap of hybridized 2D topological thin-film states are schematically shown in Fig. 3(c). The velocity $v$ of $1 \mathrm{D}$ excitations (red) is asymptotically the same as those of the gapped and hybridized 2D surface excitations (blue).

\section{B. Eigenvectors and helicity of the step states}

The four amplitudes $\mathbf{a}_{\lambda}$ of the wave function in Eq. (12) are obtained from Eqs. (15) and (16) and the condition that $\left\langle\mathbf{a}_{\lambda}^{\dagger} \mid \mathbf{a}_{\lambda}\right\rangle=1$. They are the same on both sides, $\mathrm{L}$ and R. For the two orthogonal states corresponding to $\hat{E}_{ \pm}\left(k_{x}\right)= \pm k_{x}$, we obtain

$$
\mathbf{a}_{+}=\frac{1}{2}\left(\begin{array}{c}
1 \\
i \\
-\tau_{R L} \\
i \tau_{R L}
\end{array}\right), \quad \mathbf{a}_{-}=\frac{1}{2}\left(\begin{array}{c}
1 \\
-i \\
\tau_{R L} \\
i \tau_{R L}
\end{array}\right) .
$$

The complete normalized wave functions of the two nondegenerate step states are finally given by

$$
\phi_{ \pm}\left(k_{x}, y\right)=\frac{1}{v_{0}} \mathbf{a}_{ \pm}\left[e^{\kappa_{L} y} \Theta(-y)+e^{-\kappa_{R} y} \Theta(y)\right] e^{i k_{x} x},
$$

where the normalization is $v_{0}=L_{0}^{\frac{1}{2}}\left(\kappa_{L}^{-1}+\kappa_{R}^{-1}\right)$, with $L_{0}$ denoting half the step length in the $x$ direction and $\kappa_{L, R}$ corresponding to the two possible cases in Eq. (19). Examples of the step wave functions for various integer QL thickness pairs $\left(d_{R}, d_{L}\right)$ are given in Fig. 4(a). These wave functions are eigenstates of the helicity operator. Since for the step states $\mathbf{k}=k_{x} \hat{\mathbf{x}}$, the latter is given by $\kappa_{z}=-\sigma_{y} \frac{1}{2}\left(1+\alpha_{z}\right)+$ $\sigma_{y} \frac{1}{2}\left(1-\alpha_{z}\right) \equiv-\sigma_{y}^{T}+\sigma_{y}^{B}$. The spinors $\mathbf{a}_{ \pm}$(and also the total wave functions $\phi_{ \pm}$) then fulfill $\kappa_{z} \mathbf{a}_{ \pm}=\mp \mathbf{a}_{ \pm}$. This property is inherited from the isolated T and B film states. Because of the 1D character of step states the spin is always locked perpendicular to $\mathbf{k}=k_{x} \hat{\mathbf{x}}$, i.e., parallel to $y$. It is also useful to consider the expectation values of the spin $\sigma$. One finds $\left\langle\mathbf{a}_{ \pm}\left|\sigma_{y}^{T}\right| \mathbf{a}_{ \pm}\right\rangle= \pm \frac{1}{2}$ and $\left\langle\mathbf{a}_{ \pm}\left|\sigma_{y}^{B}\right| \mathbf{a}_{ \pm}\right\rangle=\mp \frac{1}{2}$. All other spin expectation values vanish. The eigenvectors $\mathbf{a}_{ \pm}$define the field operators $\Psi_{\lambda}(x, y)(\lambda= \pm)$ of helical step states according to

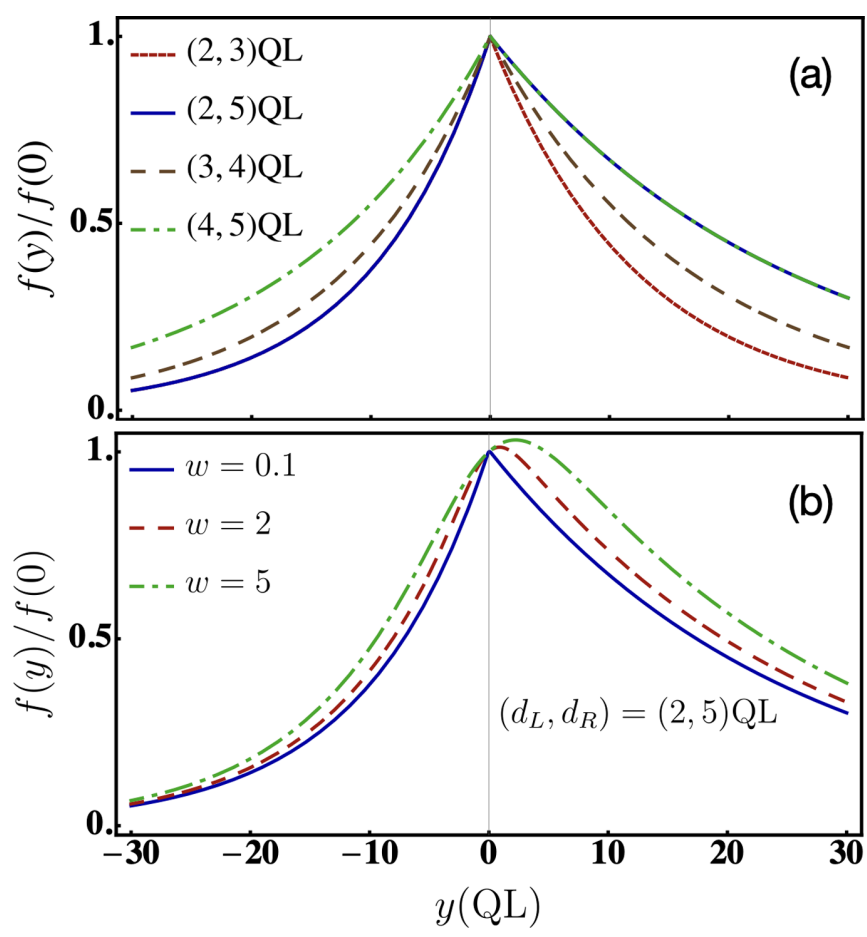

FIG. 4. Step state envelope function $f(y)$ [Eq. (28)]. (a) For different thicknesses $d_{R}$ and $d_{L}$ and a sharp step $(w=0)$. In this case the ordinate is also equal to $\left|\phi_{ \pm}\left(k_{x}, y\right)\right| /\left|\phi_{ \pm}\left(k_{x}, 0\right)\right|$ [Eq. (22)]. The pair $\left(d_{R}, d_{L}\right)=(2,3)$ is not far from the symmetric step state. (b) For fixed thickness pair $(2,5)$ but different step widths tuned by $w(\mathrm{QL})$. 
(now using $k=k_{x}$ for 1D states)

$$
\begin{aligned}
\Psi_{\lambda}(x, y) & =\sum_{k} \phi_{\lambda}^{\dagger}(k, y) \Psi_{k} \\
& =\sum_{k} \chi_{k \lambda} \frac{1}{v_{0}}\left[e^{\kappa_{L} y} \Theta(-y)+e^{-\kappa_{R} y} \Theta(y)\right] e^{i k x},
\end{aligned}
$$

where the quasiparticle operator algebra for the 1D helical step states is defined by $\chi_{k \lambda}=\mathbf{a}_{\lambda}^{\dagger} \Psi_{k}$, which is explicitly given by [using the abbreviation $\tau=\tau_{R L}=\operatorname{sgn}\left(\hat{t}_{R}-\hat{t}_{L}\right)$ in Eq. (21)]

$$
\begin{aligned}
& \chi_{k+}=\frac{1}{2}\left(c_{k \uparrow}^{T}-i c_{k \downarrow}^{T}-\tau c_{k \uparrow}^{B}-i \tau c_{k \downarrow}^{B}\right), \\
& \chi_{k-}=\frac{1}{2}\left(c_{k \uparrow}^{T}+i c_{k \downarrow}^{T}+\tau c_{k \uparrow}^{B}-i \tau c_{k \downarrow}^{B}\right) .
\end{aligned}
$$

They fulfill the canonical anticommutation relations $\left\{\chi_{k \lambda}, \chi_{k^{\prime} \lambda^{\prime}}^{\dagger}\right\}=\delta_{k k^{\prime}} \delta_{\lambda \lambda^{\prime}}$. In terms of these 1D quasiparticle operators the 1D step state Hamiltonian may be written as [see Eq. (20)]

$$
H_{S T}=\sum_{k \lambda} E_{k \lambda} \chi_{k \lambda}^{\dagger} \chi_{k \lambda}
$$

These step states form the basis to construct Majorana end states through the proximity effect originating from the superconducting substrate. Before this, however, we consider the situation for a more realistic step profile.

\section{Extension to bound states for soft steps}

The existence of the $1 \mathrm{D}$ step states is not tied to having a sharp step. A softer profile serves the same purpose, for example, replacing Eq. (8) with a soft step of width $w$ :

$$
\hat{t}(y)=\frac{1}{2}\left(\hat{t}_{R}+\hat{t}_{L}\right)+\frac{1}{2}\left(\hat{t}_{R}-\hat{t}_{L}\right) \tanh \frac{y}{w} \begin{cases}\hat{t}_{L}, & y \gg w, \\ \hat{t}_{R}, & y \ll w .\end{cases}
$$

To solve the wave Eq. (10) we now make a smooth envelope function ansatz instead of separating the $\mathrm{L}$ and $\mathrm{R}$ regimes [Eq. (12)]. It may be written as

$$
\phi_{\lambda}\left(k_{x}, y\right)=\frac{1}{v} \mathbf{a}_{\lambda} e^{i k_{x} x} f(y),
$$

with

$$
\begin{aligned}
f(y) & =\exp \left[-\tau_{R L} \int_{0}^{y} \hat{t}\left(y^{\prime}\right) d y^{\prime}\right] \\
& =\exp \left[-\tau_{R L}\left(\overline{\hat{t}} y+\hat{t}^{\prime} w \ln \cosh \frac{y}{w}\right)\right] .
\end{aligned}
$$

The form of spinors in Eq. (28) and the dispersions are the same as for the model with a sharp step. Here we define the symmetrized expressions $\overline{\hat{t}}=\frac{1}{2}\left(\hat{t}_{R}+\hat{t}_{L}\right)$ and $\hat{t}^{\prime}=\frac{1}{2}\left(\hat{t}_{R}-\hat{t}_{L}\right)$. A comparison of step and envelope functions for various widths $w$ is presented in Fig. 4(b).

\section{Influence of the $2 D$ warping term}

Our investigation of step states is based on the underlying assumption that the influence of higher-order warping terms on the $2 \mathrm{D}$ surface states can be neglected in the 1D step state formation. First, how to include them in the surface state formation of thin films where they modify the isotropic Dirac cones and circular Fermi surface into cones and a Fermi surface with a six-pronged snowflake shape is well known. A complete theory for homogeneous thin films including the lowest-order Dirac term [Eq. (1)], the warping term, and the intersurface hybridization on the same footing was developed in Ref. [21], leading to gapped and warped Dirac cones for the $2 \mathrm{D}$ thin-film quasiparticles. The remaining question of importance here is how the warping will influence the formation of the 1D step states. We may understand this in a straightforward way by treating the warping as a perturbation (which vanishes for $k_{x} \rightarrow 0$ ). In the inhomogeneous film geometry the warping term in the spin-surface presentation is given by

$$
\hat{h}^{w}\left(k_{x}, y\right)=\lambda D_{y}\left(k_{x}\right) \sigma_{z} \alpha_{0}, \quad D_{y}\left(k_{x}\right)=k_{x}\left(k_{x}^{2}+3 \partial_{y}^{2}\right),
$$

which has to be added to the unperturbed Hamiltonian in Eq. (9). Here $\lambda$ is the warping parameter (for realistic values in TIs see Ref. [21]). Then, using the 1D step wave functions in Eq. (22), the correction to the 1D step state dispersion (20) is given by

$$
\delta E_{k_{x} \pm}=\int d y \phi_{ \pm}^{\dagger}\left(k_{x}, y\right) \hat{h}^{w}\left(k_{x}, y\right) \phi_{ \pm}\left(k_{x}, y\right) .
$$

Writing the four component eigenvectors $\mathbf{a}_{ \pm}$in Eq. (21) in terms of the obvious two-component parts $\mathbf{a}_{ \pm}^{T}=\left(\boldsymbol{\alpha}_{ \pm}^{T}, \boldsymbol{\beta}_{ \pm}^{T}\right)$, we find that the expectation values $\boldsymbol{\alpha}_{ \pm}^{\dagger} \sigma_{z} \boldsymbol{\alpha}_{ \pm}=0$ and $\boldsymbol{\beta}_{ \pm}^{\dagger} \sigma_{z} \boldsymbol{\beta}_{ \pm}=$ 0 , and therefore, the warping correction to the 1D step state dispersion $\delta E_{k_{x} \pm}=0$ vanishes. We conclude that at first order in the warping scale $\lambda$ the $1 \mathrm{D}$ step state Hamiltonian Eq. (25) is unchanged; therefore, we can expect that the possible appearance of Majorana end states in the SC case as discussed in the next section is also unaffected by the warping term in this order for all momenta $k=k_{x}$. Furthermore, in the limit $k \rightarrow 0$ the warping perturbation effect vanishes intrinsically.

\section{MAJORANA ZERO MODES IN THE SC PROXIMITY-INDUCED GAP OF 1D STEP STATES}

In each of the two 1D bands of quasiparticles confined to the step the helical spin locking is protected since it is inherited from the 2D topological surface states. Therefore, they may be considered spin-locked 1D excitations. If they open a superconducting gap originating from the proximity effect of a superconducting substrate, it is natural to expect [30-36] the possible creation of Majorana zero modes (MZMs) at the end of the step line which would lead to a zero-bias conductance peak in transport and tunneling experiments $[27,37,38]$. Due to the locking of opposite spins in the helical state an underlying conventional singlet or $s$ wave superconductor with gap $\Delta_{0}$ may be used instead of the difficult-to-realize $p$-wave superconductor necessary in spinless models $[25,30,36]$. The topological state of a general 1D superconducting fermionic system is given by a topological invariant $[32,39] \mathcal{M}=(-1)^{v}$, where $v$ is the number of Fermi points including band degeneracy in one half of the Brillouin zone (BZ) for the normal state. For the considered model [Eq. (20)] with one nondegenerate band we have $v=1$, and then $\mathcal{M}=-1$ characterizes the nontrivial topology of the superconducting state which may host Majorana zero modes as end states of the step. They are described by zero-energy solutions of Bogoliubov-de Gennes (BdG) equations inside 
the SC gap and are characterized by quasiparticle operators which are identical to their conjugates. Previous scenarios to create Majorana states have mostly involved TI wires [25,26] on top of an $s$-wave superconductor and a flux passing through them to obtain the constituent 1D excitations from which Majorana states are formed. The present proposal is comparatively simple; it just takes the stepped interface between an $s$-wave superconductor and a TI with the step playing the role of the flux penetrated wire.

\section{A. Model for 1D BdG Hamiltonian of step states}

Due to the proximity effects the spin-singlet pairs of the substrate can propagate a certain distance into the normal state of the TI which is governed by the coherence length of the latter [40]. In the purely normal state it is given by $\xi_{n}=\left(\hbar v / 2 k_{B} T\right)$, which becomes large for low temperatures, so that one may expect a still sizable $s$-wave gap $\Delta_{0}^{\prime}<\Delta_{0}$ on the $\mathrm{T}$ and $\mathrm{B}$ surfaces of the topological-insulator thin film. In order to formulate the effective BCS Hamiltonian for the 1D step states, however, we have to be aware that the proximity effect works on all 2D TI surface states. Therefore, we first express the 1D Cooper pair operators formed from Eq. (24) by the $2 \mathrm{D}$ operator basis. We must keep in mind that the two $(\lambda= \pm) 1 \mathrm{D}$ dispersions fulfill $E_{-k \lambda}=-E_{k \lambda}=E_{k-\lambda}$; therefore, only interband pairing of states with opposite momenta and approximately equal energy is possible. For those pairs we have

$$
\chi_{k+} \chi_{-k-}=\frac{i}{4} \sum_{\alpha=T, B}\left(c_{k \uparrow}^{\alpha} c_{-k \downarrow}^{\alpha}-c_{k \downarrow}^{\alpha} c_{-k \uparrow}^{\alpha}\right)=-\chi_{k-} \chi_{-k+} .
$$

This means the interband pairing of spin-locked 1D quasiparticles naturally results from the proximity-induced spinsinglet pairing of 2D surface states. Here we neglected on the right side (i) triplet terms with equal amplitude since they cannot be induced by the $s$-wave substrate and (ii) inter-(T, B) surface pairing of 2D helical states which is difficult to justify on the basis of the proximity effect. Then the pair amplitude is given by

$$
\begin{aligned}
\left\langle\chi_{k+} \chi_{-k-}\right\rangle & =-\left\langle\chi_{k-} \chi_{-k+}\right\rangle \\
& =\frac{i}{4} \sum_{\alpha=T, B}\left\langle c_{k \uparrow}^{\alpha} c_{-k \downarrow}^{\alpha}-c_{k \downarrow}^{\alpha} c_{-k \uparrow}^{\alpha}\right\rangle \\
& \sim \frac{i}{4}\left(\Delta_{T}+\Delta_{B}\right)=i \Delta_{0}^{\prime},
\end{aligned}
$$

where $\Delta_{T, B}$ are the $s$-wave order parameters on the two TI surfaces introduced by the proximity effect. If $\xi_{n} \gg d$, they may be almost equal. The proximity-induced superconducting pair potential for the 1D surface states is then

$$
\begin{aligned}
\mathcal{H}_{S C}= & i \Delta_{0}^{\prime}\left[\chi_{k+} \chi_{-k-}-\chi_{k-} \chi_{-k+}\right] \\
& -i \Delta_{0}^{\prime *}\left[\chi_{-k-}^{\dagger} \chi_{k+}^{\dagger}-\chi_{-k+}^{\dagger} \chi_{k-}^{\dagger}\right]
\end{aligned}
$$

Introducing the Nambu spinors for the two 1D bands according to $\chi_{k}^{\mathrm{tr}}=\left(\chi_{k+}, \chi_{k-}, \chi_{-k+}^{\dagger}, \chi_{-k-}^{\dagger}\right)$ and adding the normal quasiparticle part in Eq. (25), we obtain for the total 1D step state BCS Hamiltonian

$$
\begin{aligned}
& \mathcal{H}=\mathcal{H}_{S T}+\mathcal{H}_{S C}=\sum_{k} \chi_{k}^{\dagger} \tilde{h}_{k} \chi_{k}, \\
& \tilde{h}_{k}=(v k-\mu) \lambda_{z} \tau_{0}-\lambda_{y}\left(\operatorname{Re} \Delta_{0}^{\prime} \tau_{x}+\operatorname{Im} \Delta_{0}^{\prime} \tau_{y}\right) .
\end{aligned}
$$

Here the $\lambda$ and $\tau$ Pauli matrices act in the space of 1D bands $( \pm)$ and Nambu particle-hole space, respectively. For simplicity we first set $\mu=0$; that is, the chemical potential lies at the Dirac point in Fig. 3; the case for general $\mu$ is treated at the end of this section. The explicit matrix form of $\tilde{h}_{\mathbf{k}}$ is shown below. The step 1D quasiparticle energies in the superconducting state are then given by

$$
\tilde{E}_{k}= \pm\left[(v k)^{2}+\left|\Delta_{0}^{\prime}\right|^{2}\right]^{\frac{1}{2}}
$$

where, generally, $\left|\Delta_{0}^{\prime}\right|<\left|t_{L}\right|,\left|t_{R}\right|$; that is, the SC gap is inside the larger 2D hybridization gap, except when $d$ is close to nodal points of $t_{L, R}(d)$ [Fig. 2(a)].

\section{B. MZM in-gap states at the step ends}

Now we will search for zero-energy solutions of the BdG Hamiltonian inside the SC gap whose wave function has to be located within the step length $-L_{0} \leqslant x \leqslant L_{0}$, where $\Delta_{0}^{\prime}$ is finite. Replacing $k \rightarrow-i \partial_{x}$ in the above Hamiltonian, we obtain the equation

$$
\left[v \lambda_{z} \tau_{0} \partial_{x}-i \lambda_{y}\left(\operatorname{Re} \Delta_{0}^{\prime} \tau_{x}+\operatorname{Im} \Delta_{0}^{\prime} \tau_{y}\right)\right] \tilde{\boldsymbol{\phi}}(x)=0 .
$$

In the space of four-dimensional Nambu spinors $\chi_{k}$ the realspace BdG Hamiltonian in Eq. (36) is represented as the matrix

$$
\tilde{h}(x)=\left(\begin{array}{cccc}
-i v \partial_{x} & 0 & 0 & i \Delta_{0}^{\prime *} \\
0 & i v \partial_{x} & -i \Delta_{0}^{\prime *} & 0 \\
0 & i \Delta_{0}^{\prime} & -i v \partial_{x} & 0 \\
-i \Delta_{0}^{\prime} & 0 & 0 & i v \partial_{x}
\end{array}\right) .
$$

Aside from a relative sign this matrix consists of two identical blocks due to the relation between interband pairings given in Eq. (31) and neglecting the intraband pairs. Using this matrix form, which factorizes into two blocks, the BdG equation [Eq. (36)] may be solved by the ansatz of Eqs. (38) and (39) assuming $\Delta_{0}^{\prime}(x)=\Delta_{0}^{\prime} \Theta\left(L_{0}+x\right) \Theta\left(L_{0}-x\right)$, i.e., constant $\Delta_{0}^{\prime}$ within the sample. The two solutions for each block decay exponentially along either $x$ or $-x$. This means they will be located at one of the ends of the step at $-L_{0}$ or $L_{0}$, which we call $\mathrm{d}$ (down) and $\mathrm{u}$ (up), respectively (Fig. 1). For the two blocks $A$ and $B$ we arrive at the zero-energy BdG wave functions $(v=\mathrm{u}, \mathrm{d})$

$$
\begin{aligned}
\tilde{\boldsymbol{\phi}}_{A}^{v}(x)=\mathbf{a}_{A}^{v} w_{v}(x), & \tilde{\boldsymbol{\phi}}_{B}^{v}(x)=\mathbf{a}_{B}^{v} w_{\nu}(x), \\
w_{d}(x)=C e^{\lambda\left(L_{0}+x\right)}, & w_{u}(x)=C e^{\lambda\left(L_{0}-x\right)},
\end{aligned}
$$

where $C=\left\{2 \lambda /\left[1-\exp \left(-4 \lambda L_{0}\right)\right]\right\}^{-\frac{1}{2}}$. The decay length $\lambda^{-1}$ of the end states is given by $\lambda=\frac{\left|\Delta_{0}^{\prime}\right|}{v}$, which may be written as $\lambda=\frac{1}{\pi} \frac{\left|\Delta_{0}^{\prime}\right|}{\Delta_{0}} \frac{v_{F}^{s}}{v} \xi^{-1}$, which is proportional to the inverse BCS coherence length $\xi$ of the SC substrate. Here the first factor describes the gap reduction factor due to the proximity effect, and the second one describes the ratio of Fermi velocities in the substrate $\left(v_{F}^{s}\right)$ and the TI $(v)$. Furthermore, the amplitude 
vectors of the wave functions are

$$
\begin{aligned}
& \left(\mathbf{a}_{A}^{u}\right)^{\operatorname{tr}}=\frac{1}{\sqrt{2}}(1,0,0,1), \quad\left(\mathbf{a}_{A}^{d}\right)^{\operatorname{tr}}=\frac{1}{i \sqrt{2}}(1,0,0,-1), \\
& \left(\mathbf{a}_{B}^{u}\right)^{\operatorname{tr}}=\frac{1}{\sqrt{2}}(0,1,1,0), \quad\left(\mathbf{a}_{B}^{d}\right)^{\operatorname{tr}}=\frac{1}{i \sqrt{2}}(0,1,-1,0) .
\end{aligned}
$$

The complete zero-energy wave functions in the SC gap, including the $y$ dependence from Eq. (22), are then given by

$$
\begin{aligned}
\tilde{\boldsymbol{\phi}}_{A, B}^{v}(x, y) & =\mathbf{a}_{A, B}^{v} w_{v}(x, y), \\
w_{v}(x, y) & =w_{v}(x) \frac{1}{v_{0}}\left[e^{\kappa_{L} y} \Theta(-y)+e^{-\kappa_{R} y} \Theta(y)\right],
\end{aligned}
$$

where $-L_{0} \leqslant x, y \leqslant L_{0}$. The wave functions for the $A$ and $B$ blocks are related by permutation of the two $1 \mathrm{D}$ band states according to $P \tilde{\boldsymbol{\phi}}_{A, B}^{\nu}=\tilde{\boldsymbol{\phi}}_{B, A}^{\nu}$, defined by the permutation operator $P=\lambda_{x} \tau_{0}$, which is a symmetry of the Hamiltonian. Therefore, the wave functions of the zero-energy states should also be symmetrized by taking the combination $\tilde{\boldsymbol{\phi}}_{A, B}^{v}+P \tilde{\boldsymbol{\phi}}_{A, B}^{v}$, meaning

$$
\tilde{\boldsymbol{\phi}}_{v}(x, y)=\tilde{\boldsymbol{\phi}}_{A}^{v}(x, y)+\tilde{\boldsymbol{\phi}}_{B}^{v}(x, y), \quad \mathbf{a}_{v}=\mathbf{a}_{A}^{v}+\mathbf{a}_{B}^{v} .
$$

For these symmetrized zero-energy wave functions we may construct their corresponding field operators according to

$$
\begin{aligned}
& \Psi_{u}=\int d x \tilde{\boldsymbol{\phi}}_{u}^{\dagger}(x) \boldsymbol{\chi}_{x}=\int d x w_{u}(x) \mathbf{a}_{u}^{\dagger} \boldsymbol{\chi}_{x}=\int d x w_{u}(x) \gamma_{1}, \\
& \Psi_{d}=\int d x \tilde{\boldsymbol{\phi}}_{d}^{\dagger}(x) \boldsymbol{\chi}_{x}=\int d x w_{d}(x) \mathbf{a}_{d}^{\dagger} \boldsymbol{\chi}_{x}=\int d x w_{d}(x) \gamma_{2},
\end{aligned}
$$

where we used the real-space representation $\chi_{x}=$ $\int d k e^{-i k x} \chi_{k}$. These operators satisfy the reality condition $\Psi_{u}^{\dagger}=\Psi_{u}$ and $\Psi_{d}^{\dagger}=\Psi_{d}$ and therefore represent two Majorana zero modes separated at the two ends $(d, u)$ of the step length. Their associated quasiparticle operators $\gamma_{1}$ and $\gamma_{2}$, corresponding to zero-energy states with $\mathrm{BdG}$ wave functions $\tilde{\phi}_{v}(x, y)$ in Eqs. (40) and (41), are then obtained as

$$
\begin{aligned}
& \gamma_{1}=\mathbf{a}_{u}^{\dagger} \chi_{x}=\frac{1}{\sqrt{2}}\left(\chi_{x+}+\chi_{x-}+\chi_{x+}^{\dagger}+\chi_{x-}^{\dagger}\right), \\
& \gamma_{2}=\mathbf{a}_{d}^{\dagger} \chi_{x}=\frac{i}{\sqrt{2}}\left(\chi_{x+}+\chi_{x-}-\chi_{x+}^{\dagger}-\chi_{x-}^{\dagger}\right),
\end{aligned}
$$

which fulfill the reality condition $\gamma_{i}^{\dagger}=\gamma_{i}(i=1,2)$. These quasiparticle operators for the MZMs satisfy the canonical Majorana anticommutation rules $\left\{\gamma_{1}, \gamma_{1}\right\}=\left\{\gamma_{2}, \gamma_{2}\right\}=2$ and $\left\{\gamma_{1}, \gamma_{2}\right\}=0$

The real-space density profile $p(x, y)=\mid w_{u}(x, y)+$ $\left.w_{d}(x, y)\right|^{2}$ of these SC in-gap zero-energy states is governed by three (inverse) length scales: perpendicular to the step by $\kappa_{L}\left(d_{L}\right)=\operatorname{sgn}\left(t_{L}\right) t_{L}\left(d_{L}\right) / v$ and $\kappa_{L}\left(d_{R}\right)=\operatorname{sgn}\left(t_{R}\right) t_{R}\left(d_{R}\right) / v$ to the right and left and parallel to the step by $\lambda=\left|\Delta_{0}^{\prime}\right| / v$. This means that the Majorana density profile can be quite anisotropic and change rapidly as a function of the relative TI film thicknesses $d_{L}$ and $d_{R}$ on both sides of the step. In the general case when both $t_{L}\left(d_{L}\right)$ and $t_{R}\left(d_{R}\right)$ are not located close to the zeros of the oscillatory function in Eq. (7), one has $\left|\Delta_{0}^{\prime}\right|<\left|t_{L}\right|,\left|t_{R}\right|$, and therefore, the Majorana profile is concentrated at the step with a more gradual decay along the step direction $x$ (Fig. 5).
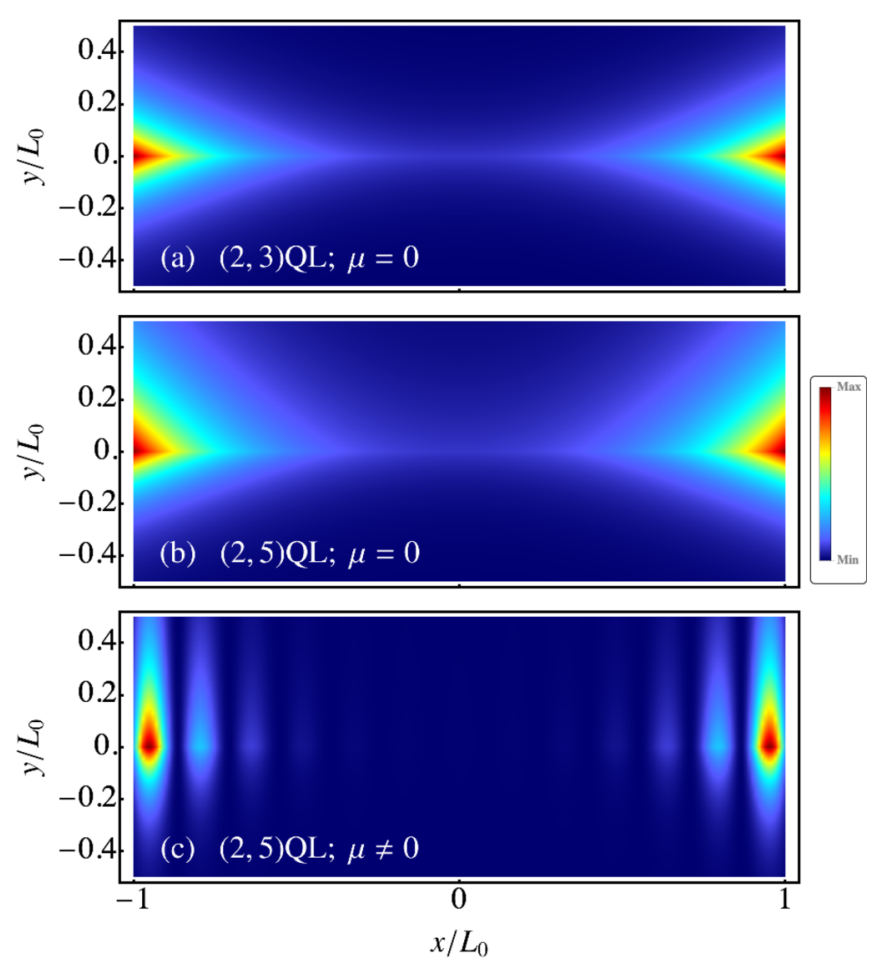

FIG. 5. Density profile $p(x, y)$ of Majorana end states (superposition of $\mathrm{u}$ and $\mathrm{d})$ on the top surface plane for the cases $\left(d_{L}, d_{R}\right)=$ $(2,3) ;(2,5)$ for chemical potential $\mu$. Asymmetry and localization degree change notably between the different pairs. For nonzero $\mu$ MZM oscillations appear according to Eq. (45). Here we set SC gap size $\left|\Delta_{0}^{\prime}\right|=0.05\left[E^{*}\right], \mu=0.25\left[E^{*}\right]$ in (c) and step length $2 L_{0}=$ $80 \mathrm{QL}$. For presentation the latter is chosen to be artificially small.

Finally, we briefly give the results for the case of the general position of the chemical potential $\mu \neq 0$ inside the hybridization gap [Fig. 3(c)], cutting the $1 \mathrm{D}$ dispersions at finite wave vector $k_{c}=\mu / v$ once in the positive half of the BZ. In the SC state this leads to quasiparticle bands

$$
\tilde{E}_{k 1,2}^{ \pm}= \pm\left[(v k \pm \mu)^{2}+\left|\Delta_{0}^{\prime}\right|^{2}\right]^{\frac{1}{2}}
$$

which now have two branches $(1,2)$ emerging from $k_{1,2}= \pm k_{c}$ and a SC gap $2\left|\Delta_{0}^{\prime}\right|$, independent of the position of the chemical potential inside the hybridization gap. The solutions for the MZM end states may be found in analogy to the above derivation. The essential difference lies in the MZM wave functions now given by

$$
\begin{aligned}
& w_{u}(x)=C e^{\lambda_{1}\left(x-L_{0}\right)} e^{i \lambda_{2} x}, \\
& w_{d}(x)=C e^{-\lambda_{1}\left(x+L_{0}\right)} e^{i \lambda_{2} x},
\end{aligned}
$$

where $\lambda_{1}=\left|\Delta_{0}^{\prime}\right| / v$ and $\lambda_{2}=\mu / v$. Thus, in addition to the exponential decay from the ends there is an oscillation of MZM form factors which becomes faster than the decay for $|\mu|>\left|\Delta_{0}^{\prime}\right|$. Analogous to Eq. (42), the self-adjoint field operators of MZM modes can be written as

$$
\begin{aligned}
& \Psi_{u}=\frac{1}{2 L_{0}} \int d x\left[w_{u}^{\prime}(x) \gamma_{1}+w_{u}^{\prime \prime} \tilde{\gamma}_{1}\right], \\
& \Psi_{d}=\frac{1}{2 L_{0}} \int d x\left[w_{d}^{\prime}(x) \gamma_{2}+w_{d}^{\prime \prime} \tilde{\gamma}_{2}\right],
\end{aligned}
$$


where we split $w_{u, d}(x)=w_{u, d}^{\prime}(x)+i w_{u, d}^{\prime \prime}(x)$ into real and imaginary parts. We have now an additional Majorana pair $\left(\tilde{\gamma}_{1}, \tilde{\gamma}_{2}\right)$ complementing Eq. (43) and defined by the quasiparticle operators

$$
\begin{aligned}
& \tilde{\gamma}_{1}=\frac{1}{i \sqrt{2}}\left(\chi_{x+}-\chi_{x-}-\chi_{x+}^{\dagger}+\chi_{x-}^{\dagger}\right), \\
& \tilde{\gamma_{2}}=\frac{1}{\sqrt{2}}\left(\chi_{x+}-\chi_{x-}+\chi_{x+}^{\dagger}-\chi_{x-}^{\dagger}\right) .
\end{aligned}
$$

The doubling is related to the existence of two Bogoliubov excitation bands for $\mu \neq 0$ arising from $k_{1,2}= \pm k_{c}$ points. Again, the Majorana anticommutation rules $\left\{\tilde{\gamma}_{1}, \tilde{\gamma}_{1}\right\}=\left\{\tilde{\gamma}_{2}, \tilde{\gamma}_{2}\right\}=2 \quad$ and $\quad\left\{\tilde{\gamma}_{1}, \tilde{\gamma}_{2}\right\}=0 \quad$ are fulfilled; furthermore, we have $\left\{\gamma_{1}, \tilde{\gamma}_{1}\right\}=\left\{\gamma_{2}, \tilde{\gamma}_{2}\right\}=0$. The density profiles are $p(x, y)=\left|w_{u}^{\prime}(x, y)+w_{d}^{\prime}(x, y)\right|^{2}$ and $\quad \tilde{p}(x, y)=\left|w_{u}^{\prime \prime}(x, y)+w_{d}^{\prime \prime}(x, y)\right|^{2}, \quad$ with $\quad w_{v}(x, y)=$ $w_{\nu}(x) \frac{1}{\nu_{0}}\left[e^{\kappa_{L} y} \Theta(-y)+e^{-\kappa_{R} y} \Theta(y)\right]$ like in Eq. (40). An example of the MZM profile for nonzero $\mu$ is shown in Fig. 5(c), which exhibits the additional oscillatory behavior.

\section{CONCLUSION AND OUTLOOK}

In this work we have shown that the helical surface states in thin films of topological insulators may be manipulated in an interesting and promising way. It was previously theoretically derived on general grounds that in thin films the surface states exhibit hybridization due to intersurface wave function overlap. This leads to a hybridization energy that both decays exponentially and oscillates with film thickness depending on materials parameters and simultaneously leads to a gap in the 2D helical surface states.

This can be exploited in a simple way by profiling the film thickness in a suitable manner, for example, by introducing a step in film thickness via the substrate such that the intersurface hybridization has opposite signs on both sides of the step. This leads to the appearance of a different type of nondegenerate $1 \mathrm{D}$ helical state confined spatially to the step with linear dispersion and again helical spin locking. These states are protected by the sign change in the hybridization, and their decay perpendicular to the step is controlled by the modulus of the hybridization energy. It should be possible to investigate the existence of these 1D step states and their dispersion by scanning tunneling microscopy (STM) spectroscopy. Before that, however, it would be useful to check by STM and ARPES experiments whether homogeneous thin films indeed exhibit the theoretically predicted and prerequisite oscillations in hybridization energy. This should immediately translate in the (rectified) oscillations of the gap size of coupled surface states. While $\mathrm{Bi}_{2} \mathrm{Se}_{3}$ does not seem to exhibit such oscilla- tions, the theoretical band parameters for $\mathrm{Bi}_{2} \mathrm{Te}_{3}$ and $\mathrm{Sb}_{2} \mathrm{Te}_{3}$ are apparently more favorable for their appearance.

If these conjectures can be experimentally verified in some cases, another highly attractive possibility opens up: When the substrate becomes a simple $s$-wave superconductor, the proximity effect leads to induced gapping of the 1D helical step states. Since the latter are nondegenerate fermions with spin locking, this can create Majorana-type zero-energy modes inside the proximity effect induced gap which are localized at the end of the steps where the gap drops to zero. Their inverse localization lengths along and perpendicular to the step are proportional to the SC gap energy and left and right intersurface hybridization energies. These Majorana end states should, like 1D step states themselves, be observable with STM spectroscopy. The present proposed scenario for creating Majorana states is exceedingly simple, requiring only a suitably profiled ( $s$-wave superconducting) substrate to create the 1D step states. Since the latter are already nondegenerate because of their helical spin-locked nature, one should not need additional arrangements like applied magnetic fields or to apply additional ferromagnetic layers which have been discussed before for the wire-type geometries for creating Majorana states.

The present simplistic geometry may be replaced by more elaborate ones. For example, the step may not be extended to the whole width $\left[-L_{0}, L_{0}\right]$ of the sample and may be present for only $|x| \leqslant x_{0}<L_{0}$. When $|x|$ approaches $x_{0}$, the thicknesses $d_{R}$ and $d_{L}$ may gradually be changed on both sides of the step to a common $d_{0}$ which satisfies $t_{R}\left(d_{0}\right)=t_{L}\left(d_{0}\right)=0$. Then the 1D helical step state will also cease to exist at the $\pm x_{0}$ position within the thin film area. This would presumably simplify investigations with the STM method and suppress unwanted effects from sample ends. Another extension would be a regular array of steps that are a certain distance $y_{0}$ apart, leading to sign change in $t(y)$ with period $2 y_{0}$. This configuration would be suitable for studying the effects of 1D step state overlap. Finally, one might form a ringlike step, i.e., a quantum dot with the proper thickness inside and outside the ring, to support a $1 \mathrm{D}$ ring state. This should lead to discretization of the linear dispersion of step states, depending on the diameter of the ring and the thickness variation characteristics. It is therefore certainly worthwhile to study these configurations and the possible in-gap (hybridization, superconducting) states and their physical consequences further.

\section{ACKNOWLEDGMENTS}

A.A. acknowledges the support of the Max Planck POSTECH/Hsinchu Center for Complex Phase Materials and financial support from the National Research Foundation (NRF) funded by the Ministry of Science of Korea (Grant No. 2016K1A4A01922028).
[1] A. A. Taskin and Y. Ando, Phys. Rev. B 84, 035301 (2011).

[2] A. A. Taskin, S. Sasaki, K. Segawa, and Y. Ando, Phys. Rev. Lett. 109, 066803 (2012).

[3] D. Hsieh, Y. Xia, D. Qian, L. Wray, J. H. Dil, F. Meier, J. Osterwalder, L. Patthey, J. G. Checkelsky, N. P. Ong, A. V.
Fedorov, H. Lin, A. Bansil, D. Grauer, Y. S. Hor, R. J. Cava, and M. Z. Hasan, Nature (London) 460, 1101 (2009).

[4] Y. L. Chen, J. G. Analytis, J.-H. Chu, Z. K. Liu, S.-K. Mo, X. L. Qi, H. J. Zhang, D. H. Lu, X. Dai, Z. Fang, S. C. Zhang, I. R. Fisher, Z. Hussain, and Z.-X. Shen, Science 325, 178 (2009). 
[5] K. Kuroda, M. Arita, K. Miyamoto, M. Ye, J. Jiang, A. Kimura, E. E. Krasovskii, E. V. Chulkov, H. Iwasawa, T. Okuda, K. Shimada, Y. Ueda, H. Namatame, and M. Taniguchi, Phys. Rev. Lett. 105, 076802 (2010).

[6] K. Hoefer, C. Becker, D. Rata, J. Swanson, P. Thalmeier, and L. H. Tjeng, Proc. Natl. Acad. Sci. USA 111, 14979 (2014).

[7] P. Roushan, J. Seo, C. V. Parker, Y. S. Hor, D. Hsieh, D. Qian, A. Richardella, M. Z. Hasan, R. J. Cava, and A. Yazdani, Nature (London) 460, 1106 (2009).

[8] T. Zhang, P. Cheng, X. Chen, J.-F. Jia, X. Ma, K. He, L. Wang, H. Zhang, X. Dai, Z. Fang, X. Xie, and Q.-K. Xue, Phys. Rev. Lett. 103, 266803 (2009).

[9] Z. Alpichshev, J. G. Analytis, J.-H. Chu, I. R. Fisher, Y. L. Chen, Z. X. Shen, A. Fang, and A. Kapitulnik, Phys. Rev. Lett. 104, 016401 (2010).

[10] Y. Okada, C. Dhital, W. Zhou, E. D. Huemiller, H. Lin, S. Basak, A. Bansil, Y.-B. Huang, H. Ding, Z. Wang, S. D. Wilson, and V. Madhavan, Phys. Rev. Lett. 106, 206805 (2011).

[11] P. Cheng, T. Zhang, K. He, X. Chen, X. Ma, and Q. Xue, Phys. E (Amsterdam, Neth.) 44, 912 (2012).

[12] Y. Kohsaka, T. Machida, K. Iwaya, M. Kanou, T. Hanaguri, and T. Sasagawa, Phys. Rev. B 95, 115307 (2017).

[13] W.-C. Lee, C. Wu, D. P. Arovas, and S.-C. Zhang, Phys. Rev. B 80, 245439 (2009).

[14] X. Zhou, C. Fang, W.-F. Tsai, and J. P. Hu, Phys. Rev. B 80, 245317 (2009).

[15] P. Rüßmann, P. Mavropoulos, and S. Blügel, Phys. Status Solidi B 258, 2000031 (2020).

[16] Y. Zhang, K. He, C.-Z. Chang, C.-L. Song, L.-L. Wang, X. Chen, J.-F. Jia, Z. Fang, X. Dai, W.-Y. Shan, S.-Q. Shen, Q. Niu, X.-L. Qi, S.-C. Zhang, X.-C. Ma, and Q.-K. Xue, Nat. Phys. 6, 584 (2010).

[17] M. Neupane et al., Nat. Commun. 5, 3841 (2014).

[18] H.-Z. Lu, W.-Y. Shan, W. Yao, Q. Niu, and S.-Q. Shen, Phys. Rev. B 81, 115407 (2010).
[19] M. M. Asmar, D. E. Sheehy, and I. Vekhter, Phys. Rev. B 97, 075419 (2018).

[20] M. M. Asmar, G. Gupta, and W.-K. Tse, arXiv:2105.12275.

[21] P. Thalmeier and A. Akbari, Phys. Rev. Research 2, 033002 (2020).

[22] J. Sau and S. Tewari, arXiv:2105.03769.

[23] J. D. Sau, S. Tewari, R. M. Lutchyn, T. D. Stanescu, and S. Das Sarma, Phys. Rev. B 82, 214509 (2010).

[24] T. D. Stanescu and S. Tewari, J. Phys.: Condens. Matter 25, 233201 (2013).

[25] A. Cook and M. Franz, Phys. Rev. B 84, 201105(R) (2011).

[26] A. M. Cook, M. M. Vazifeh, and M. Franz, Phys. Rev. B 86, 155431 (2012).

[27] A. Das, Y. Ronen, Y. Most, Y. Oreg, M. Heiblum, and H. Shtrikman, Nat. Phys. 8, 887 (2012).

[28] G. Livanas, M. Sigrist, and G. Varelogiannis, Sci. Rep. 9, 6259 (2019).

[29] W.-Y. Shan, H.-Z. Lu, and S.-Q. Shen, New J. Phys. 12, 043048 (2010).

[30] J. Alicea, Rep. Prog. Phys. 75, 076501 (2012).

[31] C. Beenakker, Annu. Rev. Condens. Matter Phys. 4, 113 (2013).

[32] S. R. Elliott and M. Franz, Rev. Mod. Phys. 87, 137 (2015).

[33] M. Sato and S. Fujimoto, J. Phys. Soc. Jpn. 85, 072001 (2016).

[34] C. Chamon, R. Jackiw, Y. Nishida, S.-Y. Pi, and L. Santos, Phys. Rev. B 81, 224515 (2010).

[35] P. Marra, D. Inotani, and M. Nitta, arXiv:2106.09047.

[36] K. Laubscher and J. Klinovaja, J. Appl. Phys. 130, 081101 (2021).

[37] S. Jeon, Y. Xie, J. Li, Z. Wang, B. A. Bernevig, and A. Yazdani, Science 358, 772 (2017).

[38] R. Aguado and L. P. Kouwenhoven, Phys. Today 73(6), 44 (2020).

[39] A. Y. Kitaev, Phys. Usp. 44, 131 (2001).

[40] P. G. de Gennes, Superconductivity of Metals and Alloys, Frontiers in Physics (Benjamin, New York, 1966). 